\title{
Transmission Electron Microscopy Study of Epitaxial Li-Mn-O Films Grown by Pulsed Laser Deposition: The Effect of Temperature on Formation of Phases
}

\author{
Haiyan Tan ${ }^{1,2^{*}}$, K. Kamala Bharathi ${ }^{1,3}$, Ichiro Takeuchi ${ }^{4}$ and Leonid A. Bendersky ${ }^{2}$ \\ 1. Institute of Materials Sciences, University of Connecticut, Storrs, CT, USA. \\ 2. Material Measurement Laboratory, National Institute of Standards and Technology (NIST), \\ Gaithersburg, MD, USA. \\ 3. Department of Physics and Nanotechnology, Research Institute, SRM University, Kattankulathur, \\ Chennai, India. \\ 4. Department of Materials Science and Engineering, University of Maryland, College Park, MD, USA. \\ * Corresponding author: Haiyan.Tan@uconn.edu
}

We demonstrate the thin film epitaxial growth of two phases, orthorhombic o- $\mathrm{LiMnO}_{2}$ and monoclinicm- $\mathrm{Li}_{2} \mathrm{MnO}_{3}$ on $\mathrm{SrRuO}_{3} / \mathrm{SrTiO}_{3}$ substrates with selected orientations at different temperatures [1]; Detailed high-resolution scanning transmission electron microscopy (HRSTEM) of cross-sections has determined the unique orientation relationship for both phases. These epitaxial films are designed to study Li-Mn-O (LMO) structural changes occurring at electrode/electrolyte interfaces, which was previously applied on $\mathrm{LiCoO}_{2}$ films [2,3].

High-resolution HAADF-STEM images of the LMO film grown at $600{ }^{\circ} \mathrm{C}$ shows coherent growth with layered structure on the SRO/STO(111) substrate (Fig. 1a,b). Furthermore, there are regions in the film that can be interpreted as belonging to $\mathrm{Li}_{0.5} \mathrm{MnO}_{2}$ spinel structure (marked as $\mathrm{S}$ in Fig, 1a,b). In some regions, the (001) layers are coherently switching their orientation and forming an orientation variant confined to a lath with parallel coherent interfaces; The nature of the layered structure was better revealed when the TEM samples were prepared with [-112]STO direction normal to the cutting plane (Fig. 1c). The image shows a contrast corresponding to a sequence along the layers of two manganese columns (two bright dots) and one lithium column (no contrast), which is of the Li--2Mn layers of m$\mathrm{Li}_{2} \mathrm{MnO}_{3}$ structure. Such contrast was shown in other microscopy studies of bulk m- $\mathrm{Li}_{2} \mathrm{MnO}_{3}[4,5]$. Stacking of the layers is not uniform and creates three alternating $60^{\circ}$-rotational variants of $\mathrm{m}-\mathrm{Li}_{2} \mathrm{MnO}_{3}$, which appears as having [110], [1-10] and [100] zone axes in Fig. 1d; this interruption of long range order is due to low-energy mistakes in packing sequence. For all three variants, assuming the same octahedral coordination of $\mathrm{Li}$ and $\mathrm{Mn}$ by oxygen, there is a common oxygen sublattice of near-close packed $a-b-c-s e q u e n c e$, the same as for the monoclinic phase.

The HAADF-STEM image of LMO film grown on SRO/STO(001) at $800{ }^{\circ} \mathrm{C}$ has a prominent zigzagging contrast of the bright $\mathrm{Mn}$ columns arrangement, which cannot be explained by the $\mathrm{m}-\mathrm{Li}_{2} \mathrm{MnO}_{3}$ structure (Fig. 2a,d,e). In contrary, the pattern fits well to the structural projection of the orthorhombic o$\mathrm{LiMnO}_{2}$ structure in [001] direction [6]. The (100) plane of o-LiMnO2 is parallel to (110) of the STO substrate. HAADF image in Fig. 2d shows in more details the arrangement of Mn columns for the [001] orientation (compare to the projected structural arrangement of $\mathrm{Mn}$ in inset). An enlarged image taken from defective regions is recognized as intergrowth of thin layers of the $\mathrm{m}-\mathrm{Li}_{2} \mathrm{MnO}_{3}$ phase, with predominately flat interphase interfaces parallel to (100)STO surface (Fig. 2e).

References:

[1] H Tan et al., Thin Solid Films 638 (2017), p. 282. 
[2] S Takeuchi et. Al., ACS Appl. Mater. Interfaces 7 (2015), p. 7901.

[3] H Tan et al., ACS Appl. Mater. Interfaces 8 (2016), p. 6727.

[4] CH Lei et al., J. Mater. Sci. 44 (2009), p. 5579.

[5] R Wang et al., Adv. Energy Mater. 3 (2013), p. 1358.

[6] IJ Davidson et al., J. Power Sources 54 (1995), p. 232.
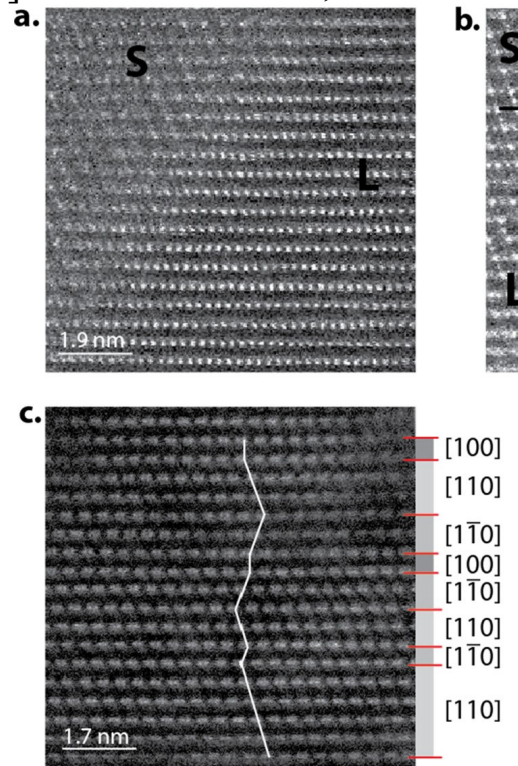

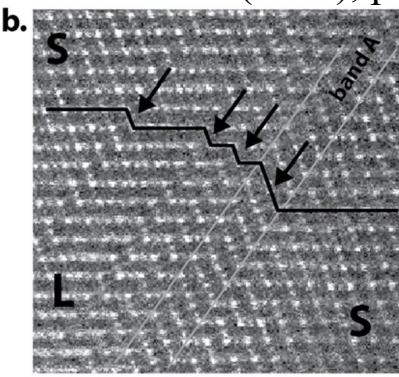

d.
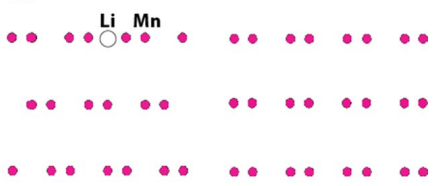

[110] [100]

Figure 1. Atomic resolved HAADF-STEM images of the LMO film grown on the SRO/STO(111) substrate at $600{ }^{\circ} \mathrm{C}$. (a, b) LMO images alone the [110]STO showing two orientational variants of the Layered phase (L) arrange in alternating bands with parallel coherent interfaces of spinel structure (S). (c) Similar STEM image from [-112]STO zone axis showing a contrast typical for the Li--2Mn layers of m-Li2MnO3. (d) structural projections of Mn columns alone [110] and [100] of m-Li2MnO3 structure.
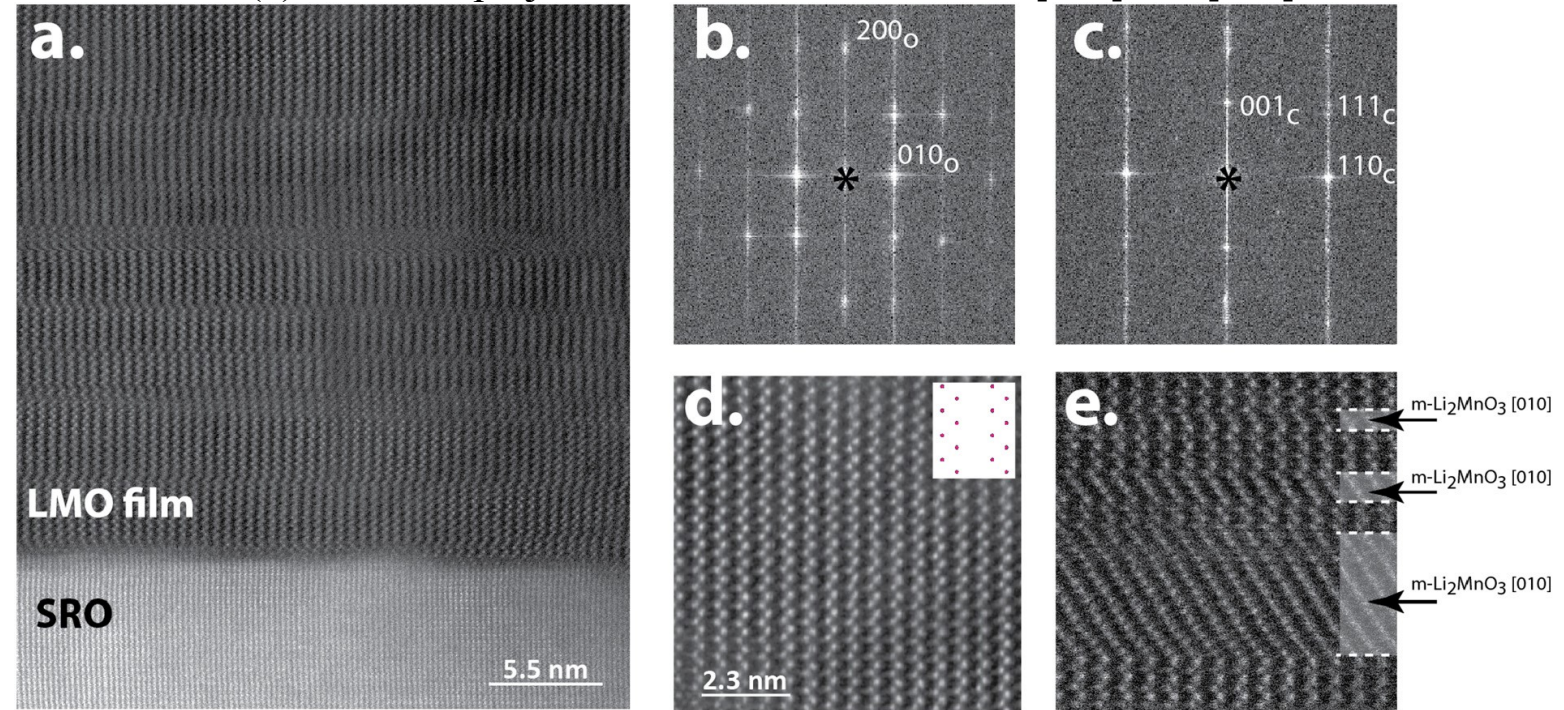

Figure 2. (a) STEM image of the LMO film grown on the SRO/STO(001) substrate at $800{ }^{\circ} \mathrm{C}$; (b, c) FFT patterns taken from the film (b) and SRO/STO substrate (c) confirm the o-LiMnO2 phase in [001] zone axis and STO in [-110] zone axis, accordingly, and (100) plane of o-LiMnO2 parallel to (110) of the substrate; (d) the region of single crystal o-LiMnO2 showing in more details a characteristic zig-zag contrast; (e) a region with defects recognized as intergrowth between o-LiMnO2 and m-Li2MnO3 phase in $[010]$ zone axis. 